\title{
Optical Frequency Comb Generation from Semiconductor Lasers Using Optical Injection and Pulsed Gain Switching
}

\author{
A. Rosado , A. Pérez-Serrano , J.M.G Tijero , A. Valle L. Pesquera and I. Esquivias
}

An Optical Frequency Comb (OFC) Generator is a laser source emitting a coherent radiation with an equally spaced group of optical frequencies. OFCs have been used in numerous fields, such as optical communications [1], RF photonics [2] and infrared spectroscopy [3]. Relatively low repetition rate OFCs (100-1000 MHz) are especially suited for dual comb spectroscopic applications. Low repetition rate OFCs from Gain-Switched (GS) semiconductor lasers using sinusoidal excitation have been reported for spectroscopic applications [4]. On the other hand, the generation of ultra-short optical pulses by pulsed excitation GS has been demonstrated [5], but as far as we know, it has not been used for OFC generation.

In this work, we consider pulsed GS in combination with optical injection (OI) to generate low repetition rate OFCs, and we demonstrate a high performance in terms of noise, flatness and bandwidth. The OFC generator is based on a master-slave configuration. The Slave Laser (SL), a Discrete Mode Laser (DML), is driven in GS operation using a combination of two signals: a bias current and a square signal provided by a pulse pattern generator, with different duty cycles and driving conditions. High-resolution spectra were analyzed with a Brillouin Optical Spectrum Analyzer (BOSA).
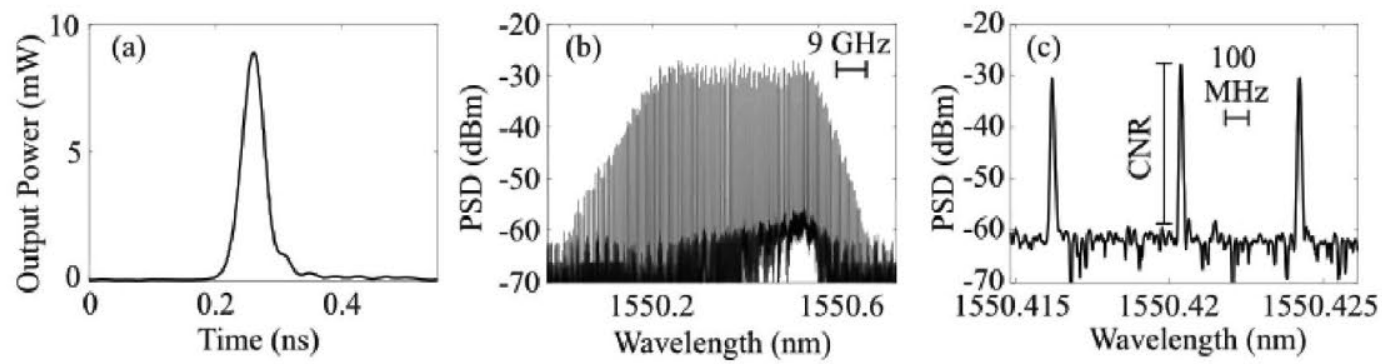

Fig. 1. Temporal trace of the output power (a) and optical spectrum (b) of the light emitted by the laser under 500 MHz pulsed electrical excitation ( $\mathrm{I}_{\mathrm{OFF}}=12.2 \mathrm{~mA}$ and $\mathrm{I}_{\mathrm{ON}}=56.2 \mathrm{~mA}$ ) and optical injection $\left(\mathrm{P}_{\mathrm{INY}}=-16 \mathrm{dBm}\right.$, detuning $\Delta v=16 \mathrm{GHz}) \cdot(\mathrm{c})$ is a zoom of (b).

Fig. 1 shows the temporal and spectral responses of a laser under pulsed excitation and optical injection. In this case, a train of electrical pulses of a width $\tau=100 \mathrm{ps}$ at a $500 \mathrm{MHz}$ repetition rate generates short (40 ps at FWHM) optical pulses (Fig. 1(a)). In the spectral domain, the resulting broad and flat OFC (Fig. 1(b)) shows a very high Carrier to Noise Ratio (CNR), around $35 \mathrm{~dB}$, and 116 tones within $10 \mathrm{~dB}$, which is an increase of a factor 15 in comparison with the best value measured at this repetition frequency with sinusoidal excitation [6]. This improvement is mainly due to the OI. Under these driving conditions, short optical pulses are also achievable without OI, however, the corresponding spectrum is continuous and does not show the clearly discernible peaks characteristic of an OFC. The laser in this case is completely switched off between pulses and each new pulse builds up from spontaneously emitted photons with random initial phase, and therefore, their optical fields do not add up coherently resulting in the absence of discrete spectral lines. However, when OI is applied, the externally injected photons are acting as the seed for the GS pulses; they provide a common phase resulting in the presence of discrete spectral lines. By optimizing the driving and optical injection conditions, we can generate high quality OFCs for a wide range of low repetition rates, including modulation frequencies lower than $100 \mathrm{MHz}$. In conclusion, GS with pulsed excitation in combination with $\mathrm{OI}$ is demonstrated as an excellent technique to generate broad and flat OFCs with a relatively low frequency spacing.

[1] M. Imran, P. M. Anandarajah, A. Kaszubowska-Anandarajah, N. Sambo, and L. Poti, "A survey of optical carrier generation techniques for terabit capacity elastic optical networks". IEEE Commun. Surv. Tutor., 20(1), 211-263, 2018.

[2] V. Torres-Company and A. M. Weiner, "Optical frequency comb technology for ultra-broadband radio-frequency photonics". Laser. Photon. Rev., vol. 8, no. 3, pp. 368-393, 2014.

[3] I. Coddington, N. Newbury, and W. Swann, "Dual-comb spectroscopy," Optica, vol. 3, p. 414, apr 2016.

[4] B. Jerez, P. Martín-Mateos, E. Prior, C. de Dios, and P. Acedo, "Dual optical frequency comb architecture with capabilities from visible to mid-infrared". Opt. Exp., 24(13), 14986-14994, 2016.

[5] S. Chen, M. Yoshita, A. Sato, T. Ito, H. Akiyama, \& H. Yokoyama, "Dynamics of short-pulse generation via spectral filtering from intensely excited gain-switched 1.55- $\mu$ m distributed-feedback laser diodes". Opt. Exp., 21(9), 10597-10605, 2013.

[6] A. Rosado, A. Pérez-Serrano, J. M. G. Tijero, Á. Valle, L. Pesquera, and I. Esquivias, "Experimental study of optical frequency comb generation in gain-switched semiconductor lasers," Opt. \& Laser Technol. 108, 542-550 (2018). 